\title{
Minimizing Energy Consumption \& Prolong Network Lifetime of Linear WSNs
}

\author{
Roshni Kapoor ${ }^{1}$, Pragya Awasthi ${ }^{2}$ \\ Dept. of Electronics \& Communication, BBD University, Lucknow, India ${ }^{1,2}$
}

\begin{abstract}
Wireless sensor networks (WSNs) have been employed as an ideal solution in many applications for data gathering in harsh environment. Energy consumption is a key issue in wireless sensor networks since nodes are often battery operated. Medium access control (MAC) protocol plays an important role in energy efficiency in wireless sensor networks because nodes' access to the shared medium is coordinated by the MAC layer. An energy efficient MAC protocol is designed for data gathering in linear wireless sensor networks. In order to enhance the performance, when a source node transmits data to the sink, Best set of nodes are selected for forwarding data according to the energy consumption factor and residual energy balance factor. Some simulation experiments are conducted and the results show that, the proposed protocol provides better energy efficiency and long lifetime than the existing SLDMAC protocol.
\end{abstract}

Keywords: WSN, Linear topology, MAC protocol, Low-energy consumption, Relay nodes.

\section{INTRODUCTION}

WSNs can be ideal solutions in many cases, such as environment monitoring, city traffic monitoring, etc. A MAC protocol is the fundament of the communication among sensor nodes, which ensures that there isn't any two nodes interfering with each other's transmission so that successful operation of the network. Many energy efficient MAC protocols have been proposed for WSN, including S-MAC, T-MAC, DMAC, etc. These protocols are similar to MAC protocols in conventional wireless networks or ad hoc networks. Although some of these have taken the features of data transmissions in WSNs, most of them are based on random deployment rather than a specific topology. In fact, linear topology is commonly used in many applications, such as power line monitoring, street lights monitoring and so on. WSN in which sensor nodes are deployed in linear topology is called linear WSN. There usually exits more hops when data transmit from the sensor nodes to the sink. However, there is few MAC protocol proposed to adapt to the characteristics of linear WSN.

In order to improve the energy efficiency and prolong the network life time, we designed an energy efficient MAC protocol, Data gathering MAC protocol for Improved Selective relay based Linear WSN (ISLDMAC), for linear WSN. Our contributions are: 1) we formulate the energy consumption of sensor nodes in a linear WSN. 2) Best set of nodes are selected by genetic algorithm on the basis of Impact factor. 3) We devised a gradient sleep/ wakeup schedule mechanism for sensor nodes in the network in which the concept of layering is adopted.

\section{RELATED WORKS}

There are many literatures on design and implementation of MAC protocols for WSN. Most of them focus on reducing energy consumption of nodes and prolong network lifetime. CMAC is proposed to avoid the cost for synchronization. DMAC is a tree based WSN data collection protocol. The layer of node in the tree is used to schedule the sleep/wakeup states of nodes. The data transmission direction is from the leaves to the root of the tree.

However it does not take the variation of network traffic into consideration. Y.Z.Zhao et.al. developed a protocol called AS-MAC into which they introduced self-adaptive schedule. Nodes are activated according the current data traffic. H. Oh et.al. designed a TDMA-based MAC protocol for timely and reliable delivery in dynamic WSN. It addresses the problem of load unbalancing between the nodes near the sink and the other nodes. X.Y.Wang et al. Proposed a cooperative MAC protocol using neighbor nodes to relay the overhearing information to deal with the channel fading.

However, these existing MAC protocols are all based on randomly deployed irregular topology. There are still few specific energy efficient MAC protocol devised for linear topology. So we focus on energy efficient MAC protocol for linear WSN. 


\section{IMPROVED SELECTIVE RELAY NODES BASED DATA GATHERING MAC PROTOCOL}

\section{1. $\quad$ Network model}

A linear wireless sensor network (as shown in Fig.1) consists of $\mathrm{n}$ sensor nodes. The nodes set is denoted as $\mathrm{V}=\{\mathrm{v} 1, \mathrm{v} 2, \cdots, \mathrm{vn}-1, \mathrm{vn}\}$ and $\mathrm{S}$ is the sink node. $\mathrm{di}, \mathrm{j}$ is the distance between any two nodes $\mathrm{vi}$ and $\mathrm{vj}$. We assume that all the nodes are homogeneous and manually precisely installed rather than randomly scattering.

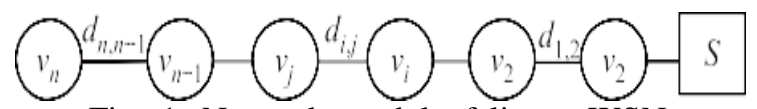

Fig. 1. Network model of linear WSN

The energy consumption of node $\mathbf{i}$ when it transmits 1 bits data to $\mathbf{j}$ can be computed by Eq.(1), and in this case the energy consumption of node $\mathbf{j}$ can be computed by Eq.(2)

$$
\begin{aligned}
& E_{t x_{i} i}=l\left(c_{1}+a d_{i, j}^{\infty}\right) \\
& E_{\pi-j}=l c_{2}
\end{aligned}
$$

Where $\mathrm{c} 1$ and $\mathrm{c} 2$ is the energy consumed per bit in the transmitter and receiver circuit respectively, a accounts for the energy dissipated in the transmit amplifier, $\alpha$ is the path loss exponent. Let $\mathrm{c}=\mathrm{c} 1+\mathrm{c} 2$, then the total energy consumption is:

$$
E=l\left(c+a d_{i, j}^{\infty}\right)
$$

Considering node $\mathrm{A}$ sends data to node $\mathrm{D}$, if $d \geq\left(c / a\left(1-2^{1-\infty}\right)\right)^{1 / / \infty}$ then there must exists a relay node $\mathrm{B}$ between node A and D that it will consume lower energy if data is forwarded by node B . More generally, in this case, we can get the approach with lowest energy consumption.

Suppose that data transferred from node A to node D will be forwarded by n-1 Relay nodes. Then the energy consumption can be represented by

$$
\begin{aligned}
& f\left(x_{1}, x_{2}, \ldots, x_{n 2}\right)=\sum_{i=1}^{n} x_{\bar{i}}^{\infty}+n c \\
& \text { Where } \sum_{i=1}^{n} x_{i}=d \text {. When } n=d /(c / a(\alpha-1))^{1 / \alpha} \text { and } d^{d}=\frac{d}{n_{0}}=(c / a(\propto-1))^{1 / \alpha}
\end{aligned}
$$

\section{The main idea}

Proper relay nodes should be chosen to forward data for conserving energy. Data transmission in linear WSN can be recognized as data gathering in a chain. Therefore, the sensor nodes can be divided in to several layers from downstream to upstream. In each round of data gathering, we can schedule the sleep/wakeup state of each node according to its layer so that each node who have data to send will wait until data from downstream arrives, then combines and sends the received data and raw data together. Those who have no data to send only need to stay in sleep state. However if the distance between adjacent nodes is too long, it would cost too much en-ergy. The basic idea of SLDMAC is to divide the whole duty cycle T into 3 phases (as shown in Fig.2.):

The basic idea of MAC is to divide the whole duty cycle T into 3 phases:

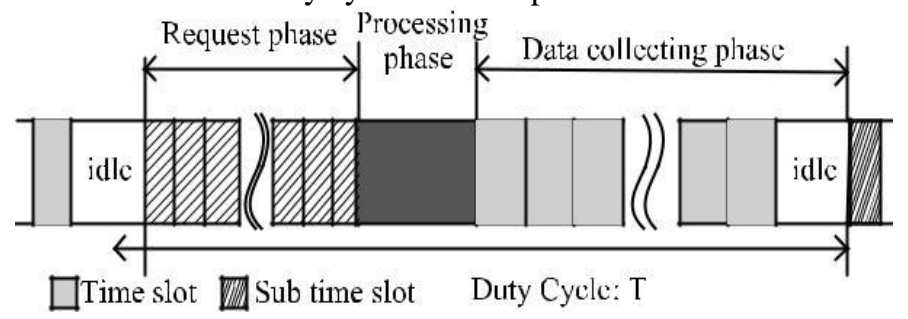

1) Request phase: It is composed of several time slots which are pre-assigned to each node. Nodes who has data to send should send a request to the sink within its own slot. The number of slots is equal to the total number of nodes. The nodes always stay in sleep until its own slot arrives and then a 1-bit request is sent to the sink. In this way, the transmitter collection Vs can be retrieved by the sink.

2) Processing phase: It is composed of two sub-phases. First, run the relay nodes selecting algorithm. Second, schedule the sleep/wakeup states for the nodes in Vs in the current duty cycle.

3) Data collecting phase: All the nodes in Vs with all the relay nodes together constitute a data gathering chain. Then, the sink node schedule the sleep/wakeup state for all the nodes on the chain to collecting sensed information. The length of this phase is the time for every nodes sending data. 
Besides, in order to synchronize the clocks of all nodes in the network, each node should transmit SYNC packets to notify the neighbors of their schedule.

\section{Selecting best set of nodes}

1. Encoding: First step towards solving problem using genetic algorithm is the encoding of solution. In this stage, phenotype is mapped to genotype. It means data is represented in genes.

2. Initialization: Take input parameter like population size, crossover probability, mutation probability, and number of generation for iteration.

3. Evaluation: Find the fitness value of each individual through fitness function.

4. Selection: Retainment of best fit individual and elimination of bad population is the task of selection.

5. Crossover: Recombination is another name for crossover of two selected parents from the pool of population.

6. Mutation: Mutation is adding new features form outside and permutation of gene within a chromosome.

7. Iterate steps 3 to 6 until terminate the loop.

8. Decoding: Decode the final solution back to phenotype.

We first analyze the impact of energy consumption by selecting different sets of node. In the case of node A sending data to node $\mathrm{D}$, it is necessary to choose a relay node $\mathrm{R}$, then $\mathrm{R}$ must be within distance of $2 \mathrm{do}$.

Suppose the distance between R and A is $\mathrm{x}$, we can carry out additional energy consumption compared to the case of do.

1. If $\mathrm{x}<\mathrm{do}$

$\Delta E=E_{x}+E_{d o-x}-E_{d 0}$

2. If $\mathrm{do}<\mathrm{x}<2 \mathrm{do}$

$\Delta E=E_{x}+E_{2 d 0-x}-2 E_{d 0}$

Suppose there are $\mathrm{k}$ nodes then residual energy of $\mathrm{k}$ nodes are

$$
E_{i}^{y}=E_{i}-\left(E_{\text {do }}+\Delta E\right)
$$

Define the residual energy factor

$$
\begin{aligned}
& \emptyset=\sum_{j=0, j \neq 1}^{k z} E_{j}-E_{a}+E_{i}^{j}-E_{a} /(k+1) \\
& \text { Where } E_{a \alpha}=\sum_{j=0, j=\bar{s}}^{k j} E_{j}+E_{i}^{s} /(k+1)
\end{aligned}
$$

A smaller $\varnothing$ indicates that the residual energy of each node is close to the average one $E_{a x}$. We also define the residual energy impact factor as $\delta=\Delta E^{2}$

Combining the residual energy balance factor and the residual energy impact factor together, we get a comprehensive impact factor so that both the residual energy and energy balance are considered.

$$
\varepsilon=\varnothing+\delta=\sum_{i=0}^{\infty} \frac{E_{j}-E_{i \alpha}^{2}}{k+1}+\Delta E^{2}
$$

choose a set of nodes with the minimum impact factor among its neighbors.

4. Gradient schedule mechanism

All the nodes in $V_{s}$ and $V_{r}$ composed a linear data gather-ing chain, they need to be wakeup during the data collecting phase. In the chain, we use the concept of layer to represent the relationship between nodes. The most adjacent node of the sink is defined as the first layer. Therefore, we set Layer 2, Layer 3, . ., Layer $\mathrm{N}$ to each node in the chain accordingly.

The data collection procedure always start from the highest level layer. Nodes on the chain are waked up only when in their turn for sending data. We note the transmitting and receiving time of each node as T. Obviously, it can be divided into two part: the receiving time RX and transmitting time TX, T $=\mathrm{RX}+\mathrm{TX}$. During RX, it receives data from a node of higher layer while during TX and sends packets which mix the received data and current sensed data together to a node of lower layer. For any two adjacent nodes of layer i and layer $\mathrm{i}-1$, their receiving and transmitting time Ti and $\mathrm{Ti}-1$ is successive. Specifically, TXi and RXi-1 are absolutely overlapped. 
The gradient schedule mechanism is shown in Fig.3. In a data gathering chain, the node with highest layer is first scheduled into wakeup state. Then other nodes are scheduled in turns according to their layers. Once the transmitting is completed, the node is scheduled into sleep state.

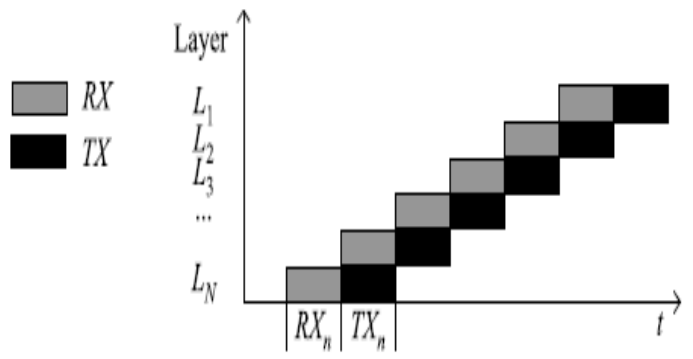

A bits vector (as shown in Fig.4) is designed as a feedback information of schedule result. Each bit in this vector represents a schedule result for a corresponding node. A ' 1 ' bit means the specific node should stay in wakeup state while a ' 0 ' bit means it should switched into sleep mode.

The index of the corresponding ' 1 ' bit for a specific node in the vector indicates its layer in the chain. This vector is encapsulated in a controlling frame. The length of the vector is set as the total number of nodes in the network. Such controlling frames is much shorter than data frames. Besides, the number of controlling frames is fairly small compared to data frames.

\section{EXPERIMENT AND ANALYSIS}

\section{Experimental setting}

The experiments are conducted on MATLAB platform. N (4-20) nodes are deployed in linear topology with length of 500 meters. The basic parameters are shown Table 1 . Further, the initial energy of each node is $0.1 \mathrm{~J}$ and the time of transmitting full load data is $\mathrm{Tu}=10 \mathrm{~ms}$. The length of data to be sends is 300 bits.

TABLE 1. Parameters and their values in our experiments

\begin{tabular}{|c|l|c|l|}
\hline Parameters & Values & Parameters & Values \\
\hline $\mathrm{C}_{1}$ & $50 \mathrm{Nj} / \mathrm{bit}$ & $\mathrm{a}$ & $10 \mathrm{Pj} / \mathrm{bit} / \mathrm{m}$ \\
\hline $\mathrm{C}_{2}$ & $50 \mathrm{Nj} / \mathrm{bit}$ & $\mathrm{d}_{0}$ & $100 \mathrm{~m}$ \\
\hline$\propto$ & 2 & & \\
\hline
\end{tabular}

The total energy consumption of the network, the network Lifetime are evaluated in our simulations.

\section{Results and analysis}

Some experiments are conducted to compare our Improved SLDMAC with SLDMAC protocol. We first evaluate the performance of the 3 protocols with increasing number of nodes. We set $\lambda=0.3$ to adapt a normal data traffic.

Fig. 1 indicates that Improved SLDMAC consumes less energy than SLDMAC no matter how many nodes there are in the network.

Because Improved SLDMAC always choose best set of nodes to forward data. When the number of nodes increases, the distance between adjacent nodes reduces and the number of nodes which have data to send increases, so the chance for choosing relay nodes became smaller. Then the performance of Improved SLDMAC compared to SLDMAC also consumes less energy. The data gathering chain in DMAC is composed of all the nodes in the network so that those who with higher layer than the source node need to keeplistening which cost much energy. With the increasing of sensor nodes, the distance between adjacent nodes may be shorter than the best forward distance so that it costs more energy than the best case. However, in SLDMAC, nodes with no data to send are not involved in the chain so that they do not need to keep listening.

Fig. 2 shows the number of nodes has little impact on Improved SLDMAC. Further, it always has longer lifetime than the SLDMAC protocol. That is because SLDMAC take both nodes' energy consumption and residual energy balance into consideration. However, DMAC do not choose relay nodes at all. Therefore, the most adjacent node of sink may run out energy first. Lifetime of LDMAC is shorter than DMAC when the number of nodes is small but longer than DMAC when the number of nodes increases. Lifetime of DMAC drops rapidly with the increasing of nodes. i.e., the number of nodes has much impact on DMAC. The most adjacent node of sink may run out energy first. 


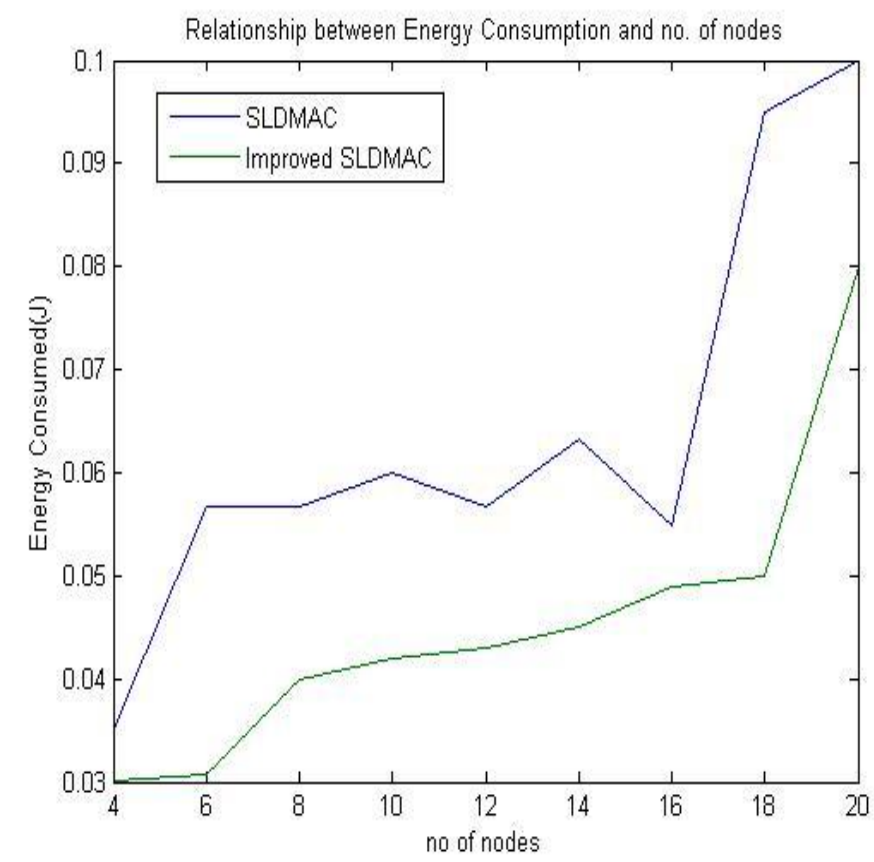

Fig 1. Comparison of Sldmac with Improved sldmac of energy consumption

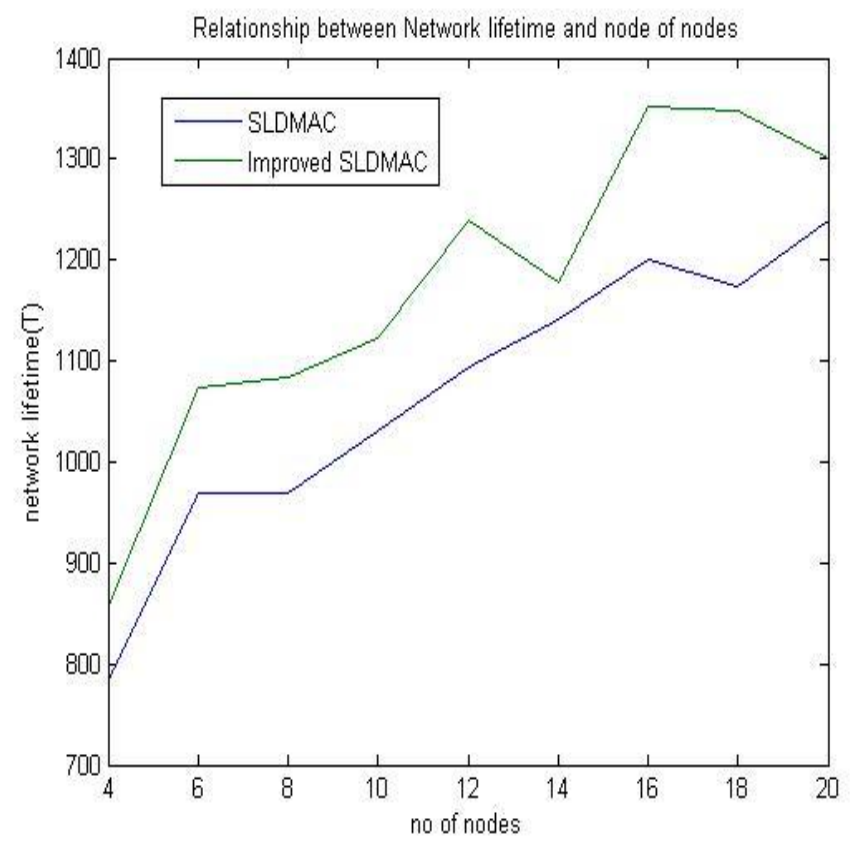

Fig 2. Comparison of Sldmac with Improved sldmac of Network lifetime

\section{CONCLUSION}

Linear WSNs have a wide variety of applications in practical. We devised a MAC protocol, Improved SLDMAC, for data gathering in linear WSNs based on best selected set of nodes. Best set of nodes are selected for energy conservation, balance the energy consumption between nodes and prolong the network lifetime consequently. We introduced node energy consumption factor and residual energy balance factor into the procedure of selecting relay nodes. A gradient nodes duty cycle schedule mechanism is proposed for this specific linear data gathering chain. The experiment results show that our proposed Improved SLDMAC has better performance than the other two protocols in the case of linear wireless sensor network. 


\section{REFERENCES}

[1] “An Energy Efficient MAC Protocol for Linear WSNs" DAI Guoyong1,2, MIAO Chunyu1, YING Kezhen1, WANG Kai1 and CHEN Qingzhang1Chinese Journal of Electronics Vol.24, No.4, Oct. 2015

[2] C. Buratti, A. Conti, D. Dardari and R. Verdone, "An overview on wireless sensor networks technology and evolution", Sensors, Vol.9, No.9, pp.6869-6896, 2009.

[3] J. Yick, B. Mukherjee and D. Ghosal, "Wireless sensor network survey", Computer Networks, Vol.52, No.12, pp.2292-2330, 2008.

[4] B. Wang, H.B. Lim and D. Ma, "A survey of movement strategies for improving network coverage in wireless sensor networks", Computer Communications, Vol.32, No.13-14, pp.1427-1436, 2009.

[5] W. Ye, J. Heidemann and D. Estrin, "An energy-efficient MAC protocol for wireless sensor networks", Proc of Twenty-First Annual Joint Conference of the IEEE Computer and Communications Societies, New York, USA, pp. 1567-1576, 2002.

[6] Y. Wei, J. Heidemann and D. Estrin, "Medium access control with coordinated adaptive sleeping for wireless sensor networks", IEEE/ACM Transactions on Networking, Vol.12, No.3, pp.493-506, 2004.

[7] M. Li, Y. Xiong, Y. Chen and H. Si, "A MAC protocol for target-tracking in wireless sensor network", Chinese Journal of Electronics, Vol.22, No.2, pp.359-362, 2013.

[8] Z. Yan, S. Min, L. Jian-dong, T. Ye, Y. Jun-liang and T. Di, "An enhanced cooperative MAC protocol for multi-hop ad hoc networks", Acta Electronica Sinica, Vol.39, No.1, pp.224-232, 2011. (in Chinese)

[9] L.K. Dam TV, "An adaptive energy-efficient MAC protocol for wireless sensor networks", Proc. of the First ACM Conference on Embedded Networked Sensor Systems, Los Angeles,CA, USA, 2003.

[10] G. Lu, B. Krishnamachari and C.S. Raghavendra, "An adaptive energy-efficient and low-latency MAC for tree-based data gathering in sensor networks", Wireless Communications and Mobile Computing, Vol.7, No.7, pp.863-875, 2007.

[11] Jawhar, N. Mohamed, K. Shuaib, and N. Kesserwan, "An efficient framework and networking protocol for linear wireless sensor networks", Ad Hoc \& Sensor Wireless Networks, Vol.7,pp.3-21, 2009.

[12] Jawhar, N. Mohamed and D.P. Agrawal, "Linear wireless sensor networks: Classification and applications", Journal of Network and Computer Applications, Vol.34, No.5, pp.1671-1682,2011.

[13] M. Zimmerling, W. Dargie and J.M. Reason, "Localized poweraware routing in linear wireless sensor networks", Proc. of the 2nd ACM International Conference on Context-Awareness for Self-Managing Systems, Sydney, Australia, pp.24-33, 2008.

[14] P. Huang, L. Xiao, S. Soltani, M.W. Mutka and N. Xi, "The evolution of MAC protocols in wireless sensor networks: A survey", IEEE Communications Surveys \& Tutorials, Vol.15, No.1, pp.101-120, 2013.

[15] Demirkol, C. Ersoy and F. Alagoz, "MAC protocols for wireless sensor networks: A survey", IEEE Communications Magazine,Vol.44, No.4, pp.115-121, 2006.

[16] B. Yahya and J. Ben-Othman, "Towards a classification of energy aware MAC protocols for wireless sensor networks", Wireless Communications and Mobile Computing, Vol.9, No.12, pp.1572-1607, 2009.

[17] S. Liu, K.-W. Fan and P. Sinha, "CMAC: An energy-efficient MAC layer protocol using convergent packet forwarding for wireless sensor networks", Proc of 4th Annual IEEE Communications Society Conference on Sensor, Mesh and Ad Hoc Communications and Networks, San Diego, CA, USA, pp.11-20, 2007.

[18] Y.Z. Zhao, M. Ma, C.Y. Miao and T.N. Nguyen, "An energy efficient and low-latency MAC protocol with adaptive scheduling for multi-hop wireless sensor networks", Computer Communications, Vol.33, No.12, pp.1452-1461, 2010.

[19] H. Oh and P.V. Vinh, "Design and implementation of a MAC protocol for timely and reliable delivery of command and data in dynamic wireless sensor networks", Sensors, Vol.13, No.10, pp.13228-13257, 2013

[20] X.Y. Wang, J. Li and F.L. Tang, "Network coding aware cooperative MAC protocol for wireless ad hoc networks", IEEE Transactions on Parallel and Distributed Systems, Vol.25, No.1, pp.167-179, 2014 